19,11

\title{
Теплоемкость и магнитокалорический эффект в манганитах $\operatorname{Pr}_{0.6} \mathrm{Sr}_{0.4} \mathrm{Mn}_{1-x} \mathrm{Fe}_{x} \mathrm{O}_{3}$
}

\author{
(C) А.Г. Гамзатов ${ }^{1}$, А.Б. Батдалов ${ }^{1}$, А.М. Алиев ${ }^{1}$, M. Ellouze ${ }^{2}$, F. Jemma ${ }^{2}$ \\ ${ }^{1}$ Институт фозики им. Х.И. Амирханова ДагНЦ РАН, \\ Махачкала, Россия \\ ${ }^{2}$ Sfax University, LAMMA, \\ Sfax, Tunisia \\ E-mail: gamzatov_adler@mail.ru
}

(Поступила в Редакцию 22 марта 2017 г.)

\begin{abstract}
Представлены результаты исследования теплоемкости и магнитокалорического эффекта в манганитах $\operatorname{Pr}_{0.6} \mathrm{Sr}_{0.4} \mathrm{Mn}_{1-x} \mathrm{Fe}_{x} \mathrm{O}_{3}(x=0$ и 0.1$)$ в интервале температур 80-350 К и в магнитных полях до $18 \mathrm{kOe}$. Магнитокалорический эффект оценен двумя независимыми методами: метод модуляции магнитного поля (прямой метод) и из данных теплоемкости в магнитном поле и без поля (косвенный метод). При замещении атомов $\mathrm{Mn}$ атомами $\mathrm{Fe}(x=0.1) T_{\mathrm{C}}$ смешается на $167 \mathrm{~K}$ в сторону низких температур, при этом величина МКЭ в магнитном поле $18 \mathrm{kOе}$ меняется незначительно с $\Delta S_{M}=2.05 \mathrm{~J} / \mathrm{kg} \cdot \mathrm{K}$ и $2.31 \mathrm{~J} / \mathrm{kg} \cdot \mathrm{K}$ для $x=0$ и 0.10 соответственно.
\end{abstract}

Работа выполнена в рамках государственного задания ФАНО России (№ 0203-2016-0009) с использованием оборудования Аналитического центра коллективного пользования Дагестанского научного центра РАН.

DOI: 10.21883/FTT.2017.10.44982.089

\section{1. Введение}

Несмотря на накопленный огромный экспериментальный и теоретический материал по манганитам, все еще сохраняется интерес к изучению их физических свойств, связанный с тем, что манганиты представляют собой превосходный модельный объект для исследования фундаментальных физических свойств сильно коррелированных электронных систем. Как показывают исследования, в манганитах проявляется глубокая взаимосвязь магнитной, решеточной и электронной подсистем твердого тела. K тому же оказалось, что в манганитах магнитные и обменные взаимодействия являются ведущими и определяют электронные и решеточные свойства кристалла, следствием чего является богатая фазовая диаграмма манганитов [1].

В то же время, манганиты являются яркими представителями материалов с гигантскими величинами магнитокалорического эффекта (МКЭ), что делает их потенциальными кандидатами для использования в технологии магнитного охлаждения [2-5].

В последние годы возрос интерес к исследованию физических свойств манганитов со смешанным замещением как в $A$ подрешетке, так и в подрешетке $\mathrm{Mn}$. Поскольку в этих сериях манганитов одновременно замещаются ионы в разных узлах решетки, соответственно разным будет характер влияния такого замещения на физические свойства манганитов.

Частичное замещение ионов $\mathrm{Mn}$ ионами переходных металлов $(M)$ в манганитах $\operatorname{Ln}_{1-x} A_{x} \mathrm{Mn}_{1-y} M_{y} \mathrm{O}_{3}$ приводит к изменениям отношения $\mathrm{Mn}^{3+} / \mathrm{Mn}^{4+}$ и некоторые связи $\mathrm{Mn}^{3+}-\mathrm{O}^{2-}-\mathrm{Mn}^{4+}$ заменяются на
$\mathrm{M}^{3+}-\mathrm{O}^{2-}-\mathrm{Mn}^{4+}$. Это вызывает беспорядок в механизме переноса заряда. Такое нарушение сопровождается постепенным ослаблением ферромагнетизма с заметными изменениями в магнитных и транспортных свойствах при увеличении содержания $M^{3+}[6]$.

В системе $\mathrm{Pr}_{0.6} \mathrm{Sr}_{0.4} \mathrm{Mn}_{1-x} \mathrm{Fe}_{x} \mathrm{O}_{3} \quad$ композиция $\mathrm{Pr}_{0.6} \mathrm{Sr}_{0.4} \mathrm{MnO}_{3} \quad(x=0)$ переходит в ферромагнитную фазу при $T_{\mathrm{C}}=300 \mathrm{~K}$ [7]. Замещение ионов $\mathrm{Mn}^{3+} \mathrm{c}$ ионным радиусом $r_{\mathrm{Mn}^{3+}}=0.72 \AA$ на ион другого ферромагнитного металла $\mathrm{Fe}^{3+}$ с большим ионным радиусом $r_{\mathrm{Fe}^{3+}}=0.785 \AA$ приводит к росту длин связей $\mathrm{Mn}-\mathrm{O}$, увеличению среднего ионного радиуса и, как следствие, к ослаблению ферромагнитного взаимодействия и уменьшению $T_{\mathrm{C}}$ от $300 \mathrm{~K}$ (для $x=0$ ) до $130 \mathrm{~K}$ (для $x=0.1)$ [8]. Информацию о магнитных, электрических, структурных свойствах некоторых композиций манганита $\operatorname{Pr}_{0.6} \mathrm{Sr}_{0.4} \mathrm{Mn}_{1-x} \mathrm{Fe}_{x} \mathrm{O}_{3}$ можно найти в работах [8-16].

В данной работе приводятся результаты экспериментального исследования теплоемкости $\left(C_{p}\right)$ и магнитокалорического эффекта $(\Delta T, \Delta S)$ в нанокристаллических образцах $\operatorname{Pr}_{0.6} \mathrm{Sr}_{0.4} \mathrm{MnO}_{3}$ и $\operatorname{Pr}_{0.6} \mathrm{Sr}_{0.4} \mathrm{Mn}_{0.9} \mathrm{Fe}_{0.1} \mathrm{O}_{3}$ в интервале температур 80-350 К и в магнитных полях $18 \mathrm{kOe}$.

\section{2. Образцы и эксперимент}

Образцы $\operatorname{Pr}_{0.6} \mathrm{Sr}_{0.4} \mathrm{Mn}_{1-x} \mathrm{Fe}_{x} \mathrm{O}_{3} \quad(x=0 \quad$ и 0.1$)$ были синтезированы по керамической технологии и имели однородную структуру со средним размером гранул 200-300 nm. Более подробно результаты структурных и магнитных исследований для системы $\mathrm{Pr}_{0.6} \mathrm{Sr}_{0.4} \mathrm{Mn}_{1-x} \mathrm{Fe}_{x} \mathrm{O}_{3}$ приведены в работах $[8,16]$. 
Теплоемкость измерялась методом модуляции теплового потока. Прямые измерения адиабатического изменения температуры $\Delta T_{\text {ad }}$ проводились модуляционным методом [17]. Суть метода заключается в том, что к образцу прикладывается переменное магнитное поле, которое благодаря магнитокалорическому эффекту, индуцирует периодическое изменение температуры образца. Это изменение температуры регистрируется синхронным детектором посредством дифференциальной термопары, один спай которой приклеен к исследуемому образцу. Частота переменного магнитного поля в данном эксперименте составляла $0.3 \mathrm{~Hz}$. Переменное магнитное поле $18 \mathrm{kOe}$ создавалось источником постоянного магнитного поля производства фирмы AMT\&C LLC. Данная методика позволяет регистрировать изменение температуры с точностью не хуже $10^{-3} \mathrm{~K}$ [17]. Для оценки изменения магнитной энтропии $\Delta S_{M}$ использовались данные по теплоемкости $C_{P}(T, H)$.

\section{3. Результаты и обсуждение}

На рис. 1 представлены результаты измерения теплоемкости для образцов $\mathrm{Pr}_{0.6} \mathrm{Sr}_{0.4} \mathrm{MnO}_{3}$ и $\mathrm{Pr}_{0.6} \mathrm{Sr}_{0.4} \mathrm{Mn}_{0.9} \mathrm{Fe}_{0.1} \mathrm{O}_{3}$ в интервале температур $80-350 \mathrm{~K}$ при $H=0$ и $18 \mathrm{kOe}$. Как видно из рисунка, в интервале температур $92-130 \mathrm{~K}$ для образца с $x=0.1$ и $223-307 \mathrm{~K}$ для образца с $x=0$ наблюдаются аномалии, связанные с фазовым переходом ферромагнетик-парамагнетик. По данным $C_{P}(T)$, значения критических температур соответствуют $T_{\mathrm{C}}=276 \mathrm{~K}$ и $T_{\mathrm{C}}=109 \mathrm{~K}$ соответственно для $x=0$ и $x=0.10$. Как видим, частичное замещение атомов $\mathrm{Mn}$ атомами $\mathrm{Fe}$ приводит к смещению $T_{\mathrm{C}}$ в сторону низких температур почти на $170 \mathrm{~K}$. При этом величина аномалии также уменьшается. Такое поведение аномалии при фазовом переходе ферромагнетик-парамагнетик при низких температурах является характерной для большинства магнитных материалов [18-21]. Скорее всего, уменьшение аномалии при низких температурах для образца $x=0.10$ связано c ослаблением ферромагнитного взаимодействия. Магнитное поле подавляет аномалию и смещает температуру максимума в сторону высоких температур.

На pис. $2, a, b$ приведены температурные зависимости аномальных частей теплоемкости для образцов $\mathrm{Pr}_{0.6} \mathrm{Sr}_{0.4} \mathrm{MnO}_{3}$ и $\mathrm{Pr}_{0.6} \mathrm{Sr}_{0.4} \mathrm{Mn}_{0.9} \mathrm{Fe}_{0.1} \mathrm{O}_{3}$ в нулевом магнитном поле. Задача определения аномальной части $\Delta C_{p}$ по сути сводится к выделению фононного вклада в $C_{P}$ в широком интервале температур, так как $\Delta C_{p}=C_{P}-C_{\mathrm{ph}}$ (вкладом электронной компоненты можно пренебречь). Для этого мы поступили следующим образом. Температура магнитного фазового перехода для $\mathrm{Pr}_{0.6} \mathrm{Sr}_{0.4} \mathrm{Mn}_{0.9} \mathrm{Fe}_{0.1} \mathrm{O}_{3}\left(T_{\mathrm{C}}=109 \mathrm{~K}\right)$ значительно ниже в сравнении с $T_{\mathrm{C}}=276 \mathrm{~K}$ для $\mathrm{Pr}_{0.6} \mathrm{Sr}_{0.4} \mathrm{MnO}_{3}$, поэтому можно предположить, что теплоемкость
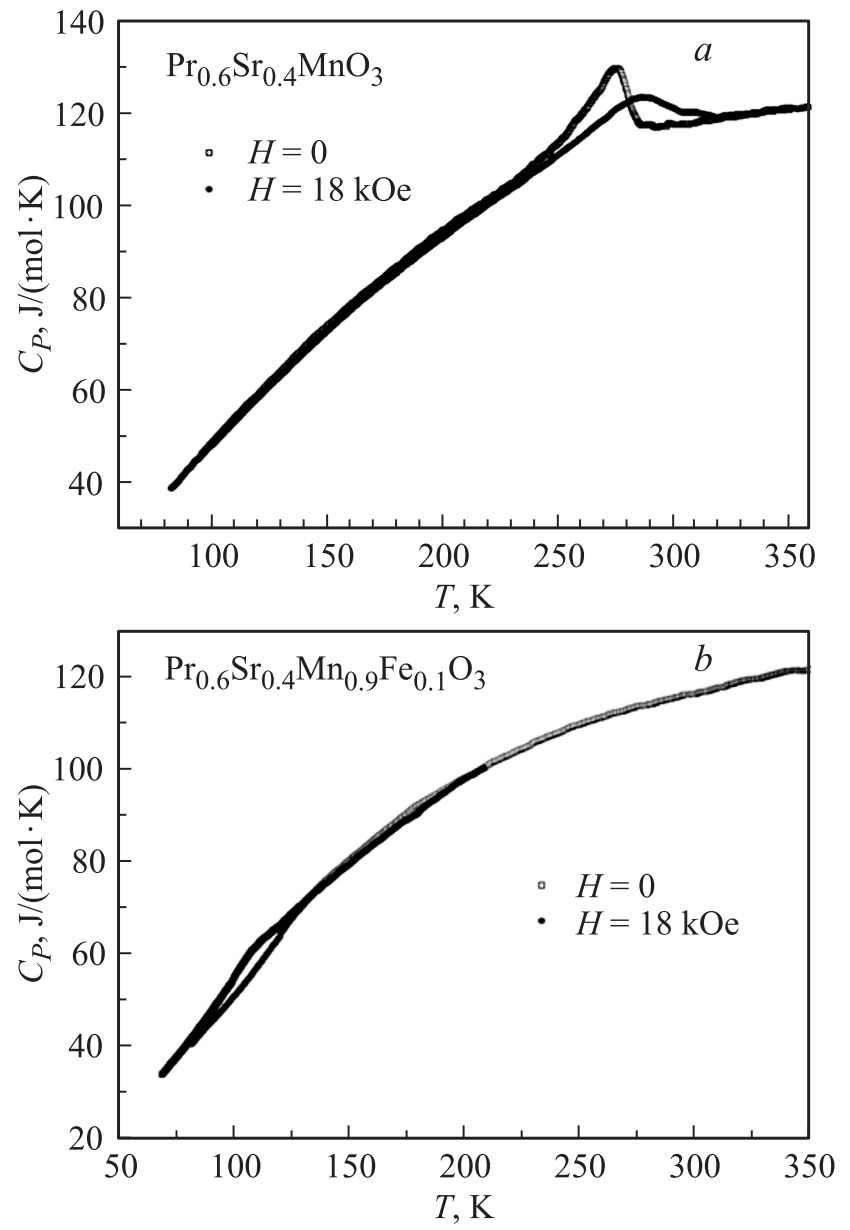

Рис. 1. Температурная зависимость теплоемкости при $H=0$ и при $H=18 \mathrm{kOe} a)$ для $\left.\operatorname{Pr}_{0.6} \mathrm{Sr}_{0.4} \mathrm{MnO}_{3}, \quad b\right)$ для $\mathrm{Pr}_{0.6} \mathrm{Sr}_{0.4} \mathrm{Mn}_{0.9} \mathrm{Fe}_{0.1} \mathrm{O}_{3}$.

$\mathrm{Pr}_{0.6} \mathrm{Sr}_{0.4} \mathrm{Mn}_{0.9} \mathrm{Fe}_{0.1} \mathrm{O}_{3}$ при $T>T_{\mathrm{C}}$ в интервале температур $150-350 \mathrm{~K}$ (т.е. в той области, где наблюдается магнитный фазовый переход в $\mathrm{Pr}_{0.6} \mathrm{Sr}_{0.4} \mathrm{MnO}_{3}$ ) носит чисто фононный характер. Аналогичным образом можно поступить и для образца $\operatorname{Pr}_{0.6} \mathrm{Sr}_{0.4} \mathrm{MnO}_{3}$. Величина скачка теплоемкости в области фазового перехода составляет $\Delta C_{p}\left(T_{\max }\right) \approx 16 \mathrm{~J} / \mathrm{mol} \cdot \mathrm{K}$ и $2.6 \mathrm{~J} / \mathrm{mol} \cdot \mathrm{K}$, соответственно для образцов $\mathrm{Pr}_{0.6} \mathrm{Sr}_{0.4} \mathrm{MnO}_{3}$ и $\mathrm{Pr}_{0.6} \mathrm{Sr}_{0.4} \mathrm{Mn}_{0.9} \mathrm{Fe}_{0.1} \mathrm{O}_{3}$. Максимальная величина аномальной части теплоемкости для образца с $x=0$ примерно в 6 раз больше чем для образца с $x=0.1$.

На рис. 2, $a, b$ также приведены температурные зависимости изменения энтропии (сплошные линии), связанные с разупорядочением магнитной системы при фазовом переходе ферромагнетик-парамагнетик, которые определялись с помощью формулы: $\Delta S(T)=$ $=\int\left(\Delta C_{p} / T\right) d T$. Значения энтропии фазового перехода для образцов $\mathrm{Pr}_{0.6} \mathrm{Sr}_{0.4} \mathrm{MnO}_{3}$ и $\mathrm{Pr}_{0.6} \mathrm{Sr}_{0.4} \mathrm{Mn}_{0.9} \mathrm{Fe}_{0.1} \mathrm{O}_{3}$ соответственно равны $1.88 \mathrm{~J} / \mathrm{mol} \cdot \mathrm{K}$ и $0.67 \mathrm{~J} / \mathrm{mol} \cdot \mathrm{K}$. Эти величины значительно меньше теоретической оценки $\Delta S=R \ln (2 S+1)=11.8 \mathrm{~J} / \mathrm{mol} \cdot \mathrm{K}$. Для монокристалли- 
Магнитокалорические свойства образцов $\operatorname{Pr}_{0.6} \mathrm{Sr}_{0.4} \mathrm{Mn}_{1-x} \mathrm{Fe}_{x} \mathrm{O}_{3}(x=0$ и 0.1$)$

\begin{tabular}{|c|c|c|c|c|c|}
\hline Образец & $T_{\mathrm{C}}, \mathrm{K}$ & $\Delta T, \mathrm{~K}$ & $\Delta S, \mathrm{~J} / \mathrm{kg} \cdot \mathrm{K}$ & $\Delta \mu H, \mathrm{~T}$ & Источник \\
\hline $\operatorname{Pr}_{0.6} \mathrm{Sr}_{0.4} \mathrm{MnO}_{3}$ & 281 & 1.10 & 2.06 & 1.8 & Данная работа \\
\hline $\mathrm{Pr}_{0.6} \mathrm{Sr}_{0.4} \mathrm{Mn}_{0.9} \mathrm{Fe}_{0.1} \mathrm{O}_{3}$ & 118 & 1.01 & 2.31 & 1.8 & $\gg \gg$ \\
\hline $\mathrm{Pr}_{0.6} \mathrm{Sr}_{0.4} \mathrm{MnO}_{3}$ & 320 & & 1.9 & 2 & {$[10]$} \\
\hline $\operatorname{Pr}_{0.6} \mathrm{Sr}_{0.4} \mathrm{MnO}_{3}$ & 305 & & 2.6 & 2 & {$[11]$} \\
\hline $\mathrm{Pr}_{0.6} \mathrm{Sr}_{0.4} \mathrm{MnO}_{3}$ & 310 & & 1.95 & 2 & {$[12]$} \\
\hline $\operatorname{Pr}_{0.6} \mathrm{Sr}_{0.4} \mathrm{MnO}_{3}$ & 306 & 1.38 & 2.7 & 2 & {$[13]$} \\
\hline $\operatorname{Pr}_{0.6} \mathrm{Sr}_{0.4} \mathrm{MnO}_{3}$ & 295 & & 2.9 & 2 & {$[15]$} \\
\hline $\mathrm{Pr}_{0.6} \mathrm{Sr}_{0.4} \mathrm{MnO}_{3}$ & 297 & & 1.55 & 2 & {$[16]$} \\
\hline $\mathrm{Pr}_{0.6} \mathrm{Sr}_{0.4} \mathrm{Mn}_{0.9} \mathrm{Fe}_{0.1} \mathrm{O}_{3}$ & 118 & & 1.85 & 2 & {$[16]$} \\
\hline $\operatorname{Pr}_{0.6} \mathrm{Sr}_{0.4} \mathrm{MnO}_{3}$ & 291 & & 2.6 & 1 & {$[22]$} \\
\hline
\end{tabular}

ческого образца $\operatorname{Pr}_{0.6} \mathrm{Sr}_{0.4} \mathrm{MnO}_{3}$ в работе [7] получено $\Delta S=3.16 \mathrm{~J} / \mathrm{mol} \cdot \mathrm{K}$. Такая разница экспериментальных данных и теоретических оценок величины энтропии перехода характерна для манганитов [12,14,18-20].

Как было сказано выше, манганиты представляют большой интерес как материалы с гигантскими значениями МКЭ, поэтому значительная часть работ по-
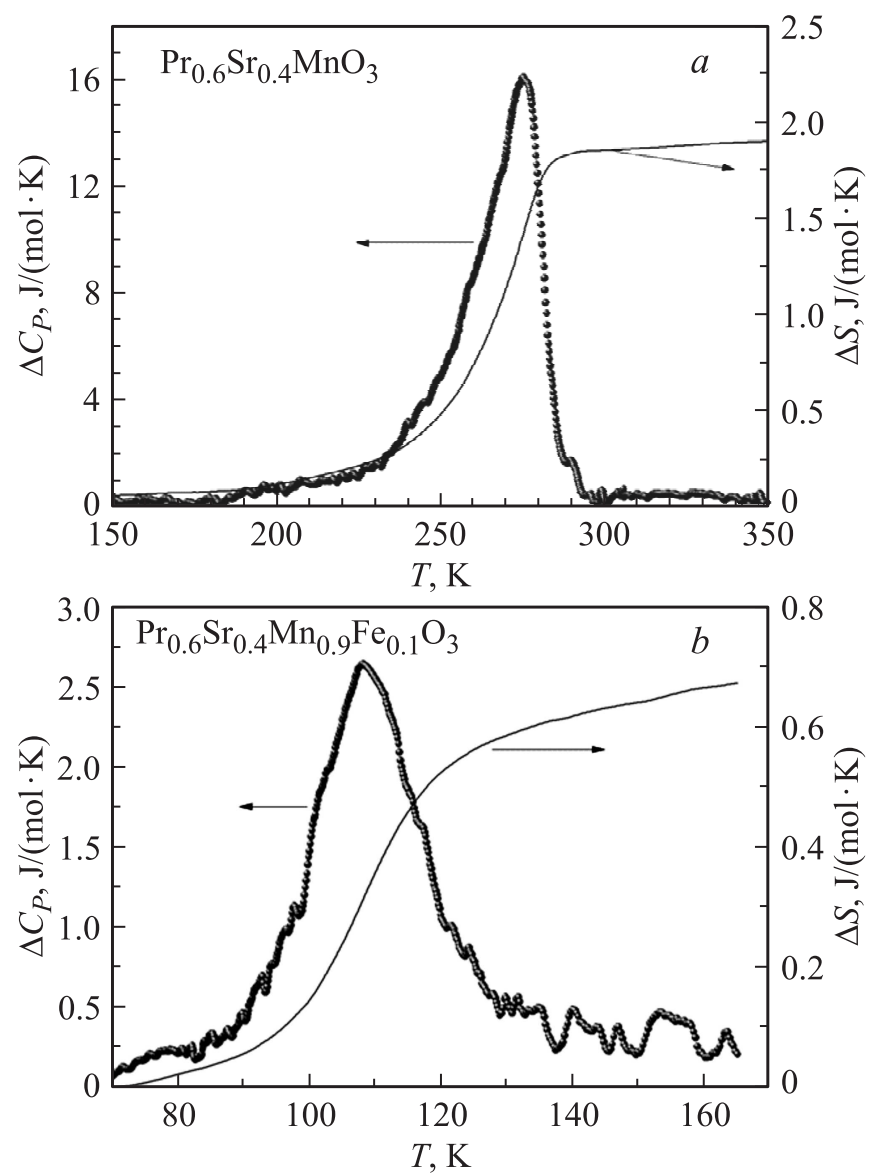

Pис. 2. Температурные зависимости аномальных частей теплоемкости для образцов $\operatorname{Pr}_{0.6} \mathrm{Sr}_{0.4} \mathrm{MnO}_{3}$ и $\mathrm{Pr}_{0.6} \mathrm{Sr}_{0.4} \mathrm{Mn}_{0.9} \mathrm{Fe}_{0.1} \mathrm{O}_{3}$ в нулевом магнитном поле. Сплошные линии температурные зависимости изменения энтропии фазового перехода. священа именно исследованию магнитокалорических свойств [10-13,15,16,22]. Величина МКЭ для одного и того же химического состава колеблется у разных авторов от $1.55 \mathrm{~J} / \mathrm{kg} \cdot \mathrm{K}$ в [16] до $2.9 \mathrm{~J} / \mathrm{kg} \cdot \mathrm{K}$ в [15] в магнитном поле $2 \mathrm{~T}$ (см. таблицу), а максимум эффекта приходится на комнатные температуры 281-320 K. Такой разброс значений, как по $\Delta S_{\max }$, так и по $T_{\mathrm{C}}$, скорее всего, связан со структурной неэквивалентностью исследуемых образцов (монокристаллы, керамика и нанокристталлы), в которых эффект от влияния границ на $\Delta S_{\max }$ будет заметно отличаться.

Как правило, магнитокалорический эффект оценивают из данных по магнитным измерениям $M(T, H)$ используя соотношение Максвелла: $\Delta S_{M}(T, \Delta H)=$ $=\int_{H_{1}}^{H_{2}}(\partial M(T, H) / \partial T)_{H} d H$. В то же время в литературе практически отсутствуют результаты прямых измерений МКЭ в этих составах. Только в работе [13] для $\operatorname{Pr}_{0.6} \mathrm{Sr}_{0.4} \mathrm{MnO}_{3}$ получено адиабатическое изменение температуры $\Delta T_{\text {ad }}=1.38 \mathrm{~K}$ в поле $2 \mathrm{~T}$ из данных теплоемкости. Магнитокалорический эффект состава $\operatorname{Pr}_{0.6} \mathrm{Sr}_{0.4} \mathrm{Mn}_{0.9} \mathrm{Fe}_{0.1} \mathrm{O}_{3}$ исследован в работе [16], $\Delta S=1.85 \mathrm{~J} / \mathrm{kg} \cdot \mathrm{K}$.

В данной работе МКЭ исследовался как прямым методом $(\Delta T)$, так и косвенным путем оценки $\Delta S_{M}$ из данных теплоемкости $C_{P}(T, H)$. На рис. 3, $a$ представлены результаты прямых измерений МКЭ в магнитном поле $18 \mathrm{kOe}$. Как видим из рисунка, в поле $18 \mathrm{kOе}$ величины $\Delta T=1.10 \mathrm{~K}$ и $1.01 \mathrm{~K}$ для $x=0$ и $x=0.1$ примерно одинаковы для обоих образцов. При этом температура максимума МКЭ для образца $x=0.1$ смещена в сторону низких температур на $167 \mathrm{~K}$. На рис. $3, b$ приведены значения изменения магнитной энтропии $\Delta S_{M}$, полученные используя данные $C_{P}(T, H)$ приведенные на рис. $1, a, b$ и используя соотношение $\Delta S_{M}=\Delta T_{\mathrm{ad}} C_{P}(T, H) / T$. Полученные значения $\Delta S_{M}=2.06 \mathrm{~J} / \mathrm{kg} \cdot \mathrm{K}$ и $2.31 \mathrm{~J} / \mathrm{kg} \cdot \mathrm{K}$ соответственно для $x=0$ и $x=0.1$. Эти значения находятся в хорошем согласии с литературными данными (см. таблицу).

Известно, что изменение энтропии $\Delta S$ может быть вычислено из данных по измерению температурных 

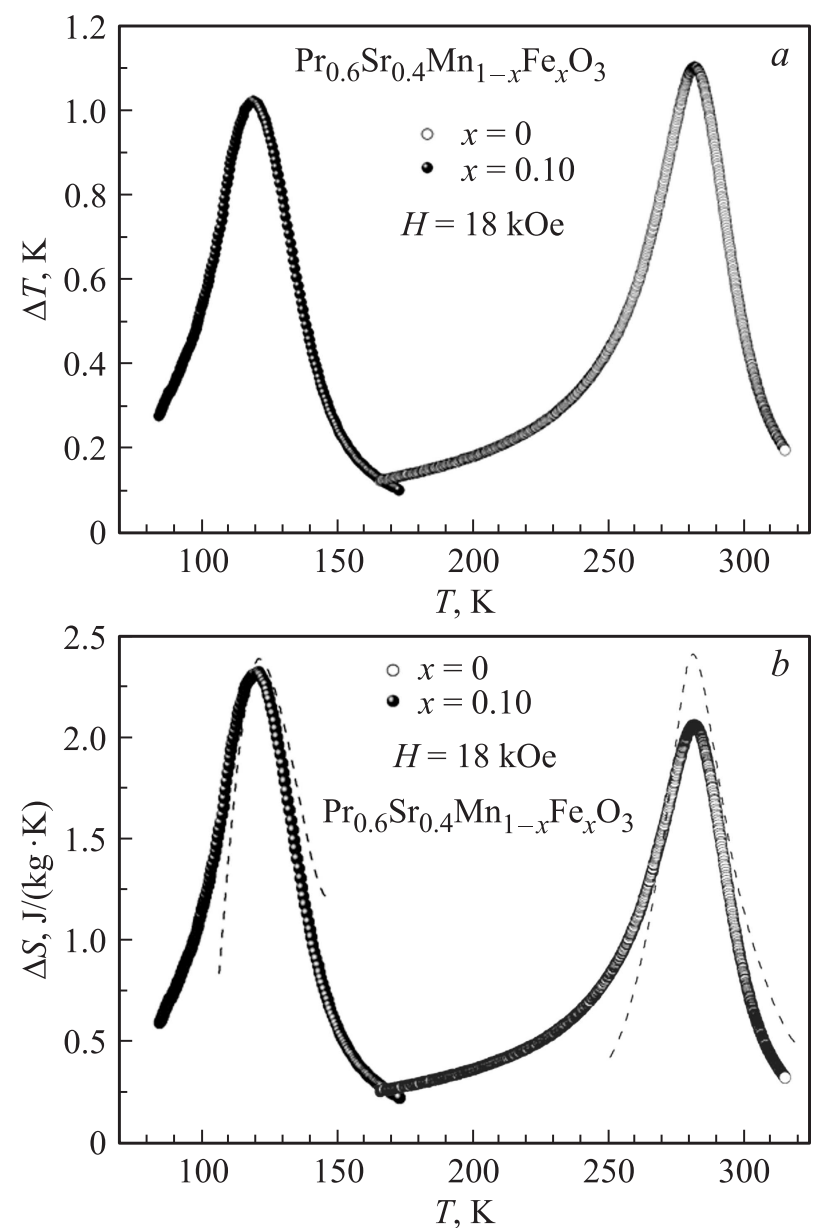

Pис. 3. a) Температурная зависимость магнитокалорического эффекта $\Delta T$ в магнитном поле $H=18 \mathrm{kOe}$ (прямые измерения). $b$ ) Температурная зависимость магнитокалорического эффекта $\Delta S$ в магнитном поле $H=18 \mathrm{kOe}$ (получены используя формулу $\left.\Delta T=\left(T / C_{H}\right) \Delta S_{H}\right)$. Штриховые линии - значения $\Delta S$, полученные с помощью формулы (1).

зависимостей теплоемкости в поле и без поля по формуле [23]

$$
\Delta S_{M}=\int_{T_{1}}^{T_{r}}\left(\frac{C_{P}\left(T, H_{0}\right)-C_{P}\left(T, H_{1}\right)}{T}\right)_{P, H} d T .
$$

Заметим, что при использовании формулы (1) погрешность в определении $\Delta S$ связана с точностью определения абсолютной величины теплоемкости и при малых значениях магнитного поля невысока. Для уменьшения погрешности при вычислении $\Delta S_{M}$ по формуле (1) необходимо провести измерения теплоемкости в сильных полях [23]. При этом значения, полученные с помощью формулы (1) хорошо согласуются с данными прямых измерений [4]. Результаты оценки магнитокалорического эффекта $\Delta S_{M}$ с помощью формулы (1) приведены на рис. $3, b$ (штриховые линии). Как видно из рисунка, величины МКЭ, оцененные с помощью формулы (1) и полученные путем прямых измерений (рис. 3,a) качественно согласуются друг с другом.

Наиболее привлекательной для системы $\mathrm{Pr}_{0.6} \mathrm{Sr}_{0.4} \mathrm{Mn}_{1-x} \mathrm{FeO}_{3}$ является слабая зависимость намагниченности $[8,16]$ и величины МКЭ от уровня допирования: несмотря на то что замещение атомов $\mathrm{Mn}$ атомами $\mathrm{Fe}(x=0.1)$ приводит к уменьшению $T_{\mathrm{C}}$ более чем на $167 \mathrm{~K}$, намагниченность и МКЭ почти не меняются. Это означает, что путем регулирования уровня замещения можно управлять $T_{\mathrm{C}}$ в широкой области температур, при этом величина МКЭ остается практически неизменной, что является важным преимуществом для магнитокалорических материалов.

\section{4. Заключение}

Таким образом в манганитах $\mathrm{Pr}_{0.6} \mathrm{SrA}_{0.4} \mathrm{Mn}_{1-x} \mathrm{FeO}_{3}$ $(x=0, x=0.1)$ исследованы теплоемкость и магнитокалорический эффект. Проведено сравнение величины МКЭ, полученного путем прямых измерений и оцененного косвенным методом (из данных теплоемкости). Полученные данные находятся в хорошем согласии друг с другом. Показано, что величина МКЭ практически не зависит от уровня замещения, а максимум смещается в область низких температур на $167 \mathrm{~K}$ при замещении атомов $\mathrm{Mn}$ атомами $\mathrm{Fe}(x=0.1)$, что позволяет управлять $T_{C}$ и областью максимума МКЭ, не меняя при этом саму величину МКЭ.

\section{Список литературы}

[1] C. Martin, A. Maignan, M. Hervieu, B. Raveau. Phys. Rev. B 60, 12191 (1999).

[2] M.H. Phan, S.C. Yu. J. Magn. Magn. Mater. 308, 325 (2007).

[3] V. Markovich, A. Wisniewski, H. Szymczak. Magnetic Properties of Perovskite Manganites and Their Modifications. Handbook of Magnetic Materials / Ed. K.H.J. Buschow. North-Holland is an imprint of Elsevier, Kidlinton, Oxford OX5 1GB, UK. (2014). V. 22. 1-201.

[4] I.K. Kamilov, A.G. Gamzatov, A.M. Aliev, A.B. Batdalov, A.A. Aliverdiev, Sh.B. Abdulvagidov, O.V. Melnikov, O.Y. Gorbenko, A.R. Kaul. J. Phys. D 40, 4413 (2007).

[5] A.M. Aliev, A.G. Gamzatov, K.I. Kamilov, A.R. Kaul, N.A. Babushkina. Appl. Phys. Lett. 101, 172401 (2012).

[6] W. Boujelben, A. Cheikh-Rouhou, M. Ellouze, J.C. Joubert. Phase Transitions 71, 127 (2000).

[7] S. Rößler, H.S. Nair, U.K. Rößler, C.M.N. Kumar, S. Elizabeth, S. Wirth. Phys. Rev. B 84, 184422 (2011).

[8] A. Nasri, S. Zouari, M. Ellouze, J.L. Rehspringer, A.F. Lehlooh, F. Elhalouani. J. Superconduct. Nov. Magn. 27, 443 (2014).

[9] P. Chen, Y.W. Du, G. Ni. Europhys. Lett. 52, 589 (2000).

[10] S. Zemni, M. Baazaoui, J. Dhahri, H. Vincent, M. Oumezzine. Mater. Lett. 63, 489 (2009).

[11] D.M. Repaka, T.S. Tripathi, M. Aparnadevi, R. Mahendiran. J. Appl. Phys. 112, 123915 (2012). 
[12] R. Thaljaoui, W. Boujelben, M. Pȩkała, K. Pȩkała, J.-F. Fagnard, P. Vanderbemden, M. Donten. A. Cheikhrouhou. J. Magn. Magn. Mater. 352, 6 (2014).

[13] M.D. Daivajna, A. Rao. Solid State Commun. 245, 65 (2016).

[14] S. Rößler, S. Harikrishnan, C.M. Naveen Kumar, H.L. Bhat, Suja Elizabeth, U.K. Rößler, F. Steglich, S. Wirth. J. Superconduct. Nov. Magn. 22, 205 (2009).

[15] D.L. Rocco, A.A. Coelho, S. Gama, M.D.C. Santos. J. Appl. Phys. 113, 113907 (2013).

[16] A. Nasri, E.K. Hlil, A.F. Lehlooh, M. Ellouze, F. Elhalouani. Eur. Phys. J. Plus 131, 110 (2016).

[17] A.M. Aliev, A.B. Batdalov, V.S. Kalitka. JETP Letters 90, 663 (2010).

[18] A.G. Gamzatov, S.B. Abdulvagidov, A.M. Aliev, A.B. Batdalov, O.V. Melnikov, O.Y. Gorbenko. JETP Letters 86, 340 (2007).

[19] A.G. Gamzatov, A.M. Aliev, A.B. Batdalov, H. Ahmadvand, H. Salamati, P. Kameli. J. Mater. Sci. 49, 294 (2014).

[20] A.M. Aliev, A.B. Batdalov, A.G. Gamzatov. Low Temperature Phys. 36, 171 (2010).

[21] L. Wang, R.-J. Wang, Y.-Y. Zhu, Z.-H. Lu, R. Xiong, Y. Liu, J. Shi. Chin. Phys. B 25, 016802 (2016).

[22] T.D. Thanh, T.A. Ho, T.V. Manh, T.L. Phan, S.-C. Yu. IEEE Trans. Magn. 50, 1 (2014).

[23] V.K. Pecharsky, K.A. Gschneidner. J. Appl. Phys. 86, 565 (1999). 